\title{
La inteligencia emocional en educación superior y militar: una revisión en América Latina ${ }^{1}$
}

\author{
Martha Patricia Vives Hurtado ${ }^{2}$ \\ Escuela de Aviación del Ejército Nacional \\ Ingrid Yuliana Arango Calderón ${ }^{3}$ \\ Escuela Militar de Cadetes "General José María Córdova” \\ Diana Carolina Contreras Gutiérrez 4 \\ Escuela de Aviación del Ejército Nacional
}

\begin{abstract}
Resumen. Este capítulo de investigación realiza una revisión de las investigaciones desarrolladas en Latinoamérica y Colombia sobre la inteligencia emocional (IE) y su importancia en entornos educativos, especialmente en educación superior y educación militar, desde el año 2004 hasta el ańo 2020. Se revisaron cincuenta artículos de las bases de datos científicas Redalyc, Scielo, Science Direct, Dialnet y algunos trabajos de grado de los repositorios de universidades. La investigación se realizó mediante un análisis documental de tipo descriptivo a partir de categorías como: conceptualización de IE, IE en entornos universitarios, IE en entornos militares e IE y personalidad. Se concluye que las
\end{abstract}

1 Este capítulo presenta los resultados del proyecto de investigación "Estrategias didácticas basadas en la inteligencia emocional como herramienta para la formación del carácter en estudiantes” del grupo de investigación en Aviación Militar de la Escuela de Aviación del Ejército (EsAvE), categorizado en C por Minciencias y con código de registro COL0077618. Los puntos de vista pertenecen a las autoras y no reflejan necesariamente los de las instituciones participantes.

2 Psicóloga de la Universidad Católica de Colombia. Magíster en Educación de la Pontificia Universidad Javeriana. Doctora en Sociología Jurídica e Instituciones Políticas de la Universidad Externado de Colombia. Investigadora de la Escuela de Aviación del Ejército Nacional. Orcid: https://orcid.org/00000001-7795-1494 - Contacto: marthaviveshurtado@cedoc.edu.co

3 Capitán del Ejército Nacional de Colombia. Especialista en ciencias militares para el planeamiento del Centro de Educación Militar. Profesional en ciencias militares y profesional en administración logística de la Escuela Militar de Cadetes "General José María Córdova”. Investigadora de la Escuela Militar de Cadetes "General José María Córdova”. Orcid: https://orcid.org/0000-0002-5995-9262 Contacto: ingridarangocalderon@cedoc.edu.co

4 Administradora ambiental de la Universidad Distrital Francisco José de Caldas. Especialista en Formulación y Evaluación de Proyectos de la Universidad Católica de Colombia. Magíster en Desarrollo y Gerencia de Proyectos de la Escuela Colombiana de Ingeniería. Investigadora de la Escuela de Aviación del Ejército Nacional (EsAVE). Orcid: https://orcid.org/0000-0002-8996-5348 - Contacto: dianacontrerasgutierrez@cedoc.edu.co 
investigaciones, en su mayoría, incluyen una revisión del origen del término, sus diferentes modelos y los autores representativos. Otras investigaciones se enfocan en la relación entre la inteligencia emocional y otras categorías, como personalidad, liderazgo, toma de decisiones, desarrollo profesional, medida o valoración de la inteligencia emocional, competencias emocionales, etc.

Palabras clave: competencias; educación; educación militar; inteligencia emocional.

\section{Introducción}

En las últimas décadas, la comunidad científica ha venido incorporando otras concepciones diferentes a la inteligencia tradicional. Las primeras pruebas evaluaban las habilidades matemáticas, lingüísticas, el racionamiento abstracto, etc., las cuales se sumaban y se establecía un coeficiente intelectual (CI). Hoy en día se habla de inteligencias múltiples, lo que ha cambiado la postura tradicional de una sola inteligencia. Esta teoría plantea que las personas pueden ser inteligentes en diferentes dimensiones de lo humano, por ejemplo, los individuos que tienen habilidades para tocar el piano o la guitarra tienen inteligencia musical; otros, pueden ser muy buenos para organizar los espacios y dibujar, teniendo inteligencia espacial, y otras personas, que son muy buenas para establecer relaciones con los demás, pues son extrovertidos, sociales, empáticos, etc., presentan inteligencia interpersonal (Gardner, 1983; citado por Antunes, 2006). Es decir, no todos somos iguales ni tenemos las mismas habilidades ni la misma inteligencia.

Otra teoría plantea que existe la inteligencia emocional (IE), comprendida como la habilidad de ser capaz de motivarse y persistir frente a las decepciones, controlar los impulsos, mostrar empatía, regular el humor, entre otras capacidades (Goleman, 1996). Esta inteligencia permite tener buenas relaciones con los demás, trabajar en equipo, ser solidarios, asertivos, etc., razón por la cual hoy en día se tiene en cuenta dentro de los procesos de selección de personal de las instituciones públicas y privadas. En consecuencia, en el sector educativo se ha estado reflexionando y trabajando en la inteligencia emocional en los procesos formativos, pues no basta con tener muchos conocimientos en un área específica y no poder trabajar en equipo, ni comprender al otro, ni controlar los estados emocionales.

Esta revisión pretende establecer el estado del conocimiento en este tema y su relación con los procesos formativos. Para tal fin, se establecieron cinco 
categorías de análisis que organizan las investigaciones encontradas y facilitan su análisis. Las categorías son: (1) conceptualización y teorización de la IE, (2) IE y entornos universitarios, (3) IE y entornos militares, (4) IE y entornos laborales, (5) IE y personalidad.

\section{Conceptualización y teorización de la inteligencia emocional}

La mayoría de las investigaciones sobre la IE profundizan en el desarrollo histórico del concepto, realizan distinciones en su definición y citan los diferentes modelos desde los autores más representativos. A continuación se describen algunas de estas investigaciones.

Bisquerra et al. (2015) plantean una definición y un modelo teórico sobre la inteligencia emocional, según el cual las competencias socioemocionales se pueden desarrollar a través de intervenciones escolares, como evidencia la eficacia que han tenido los programas de educación superior en inteligencia emocional. Por su parte, Trujillo y Rivas (2005) ahondan en el origen del concepto de IE, para lo cual hacen un recorrido por los autores que han contribuido al estudio y la medición de la inteligencia, como Gardner — con su teoría de las inteligencias múltiples_-, Salovey y Mayer, y Goleman, como creador del concepto de IE. Además, llevan a cabo una revisión de los estudios científicos y experimentales en IE y de este concepto en el campo educativo. Finalmente, ahondan en los modelos de IE en el campo administrativo, ya que un ejecutivo la requiere para su gestión, y encuentran dos tipos de modelos generales: los de habilidades y los mixtos.

En su estudio sobre la competencia emocional, Bisquerra y Pérez (2007) sostienen que esta es parte de las competencias personales. Específicamente, conceptualizan el término competencia como la capacidad de movilizar adecuadamente el conjunto de conocimientos, capacidades, habilidades y actitudes necesarias para realizar actividades diversas con un cierto nivel de calidad y eficacia. A partir de esta definición, plantean dos tipos de competencias: la socio-personal y la técnico-profesional. Posteriormente, ahondan en el concepto de competencia emocional citando a autores como Salovey y Sluyter, Goleman, Boyatzis y Mckee, Saarni, Graczyk et al. y Payton et al., así como a los institutos 
Illinois State Board of Education (IsBe), Collaborative for Academic, Social and Emotional Learning (CASEL) y State Board of Education (IsBE).

Además, se encontró otra investigación realizada por Fernández (2013), la cual hace un recorrido por la génesis de los conceptos de inteligencias múltiples e Ie con los trabajos de Broca, Binet, Galton, Cattell, Gardner, entre otros. Luego de esta descripción, el autor plantea una distinción entre inteligencia académica, inteligencia práctica, inteligencia social e inteligencias múltiples. Finalmente, presenta los antecedentes del concepto de inteligencia emocional.

Por otra parte, en la revisión teórica llevada a cabo por Bolaños et al. (2013) se aborda el origen del término inteligencia emocional en varios autores. Particularmente, mencionan a Thorndike, quien la definía como inteligencia social; a Salovey y Mayer, y Goleman, quienes la llamaron inteligencia emocional, y Gardner, con el conocido concepto de inteligencias múltiples. Con base en esta revisión, Bolańos et al. (2013) definen la IE como la capacidad de comprender emociones y conducirlas, de tal manera que se utilice para guiar la conducta y los procesos de pensamiento, para producir mejores resultados. Al final, plantean que la IE se caracteriza por los siguientes elementos: la capacidad para percibir las relaciones de forma precisa; la capacidad de aplicar las emociones para facilitar el pensamiento y el razonamiento; la capacidad para comprender las relaciones, especialmente el lenguaje de las emociones, y la capacidad para controlar las propias emociones y las de los demás.

Igualmente, Danvila y Sastre (2010) argumentan que a la IE se le ha dado mucha importancia en el desenvolvimiento de los seres humanos en los diferentes escenarios sociales y resumen las críticas que se le han hecho al concepto. Además, realizan una indagación de los diferentes conceptos relacionados con la IE, tales como: la inteligencia general, la capacidad cognitiva y la personalidad. Posteriormente, profundizan en los modelos, los instrumentos de medición y los efectos de la IE en los campos personal y laboral. Al respecto, los autores citan diversas investigaciones en las que se concluye que la IE es favorable para ambos campos, al tiempo que presentan los factores o las variables relacionadas con la IE, como la edad, el sexo y la experiencia.

Por su parte, Dueñas (2002) hace una conceptualización general del término IE a partir, sobre todo, de las investigaciones de Gardner, Mayer y Salovey, Goleman, Shapiro, Gallego et al., Cooper y Sawaf. De estos últimos, 
Dueñas (2002) destaca cuatro elementos: alfabetización emocional, agilidad emocional, profundidad emocional y alquimia emocional. Luego, la autora profundiza en la educación emocional y su importancia para atender los problemas actuales de depresión, ansiedad, violencia, drogadicción, etc., frente a lo cual sostiene que es necesario que los individuos aprendan a temprana edad a manejar sus emociones y su estabilidad emocional. Precisamente sobre ese punto, Dueñas (2002) cita a Goleman, ya que ha trabajado en la conciencia emocional, el autocontrol, la motivación, la empatía y la habilidad social. Finalmente, plantea los escenarios en el desarrollo de la educación emocional como la escuela y la familia. La autora propone algunas acciones que deben realizar los actores educativos para desarrollar la inteligencia emocional desde la infancia.

De otro lado, algunos autores han tratado de proponer modelos y relacionar los procesos educativos con el desarrollo de la IE. Por ejemplo, García y Giménez-Mas (2010) realizan una revisión de toda la literatura y plantean un modelo con dimensiones internas y externas: las primeras son la responsabilidad, el sentido común, la persuasión y la capacidad de aprender, en tanto que las segundas corresponden a la empatía, la capacidad para relacionarse, la capacidad para comunicarse, la habilidad de crear modelos mentales, la voluntad y la capacidad para adaptarse al entorno.

Extremera y Fernández (2004), por su parte, sostienen que la IE mejora el desarrollo socioemocional de los alumnos. En el mismo sentido, Fernández y Ruiz (2008) examinan las políticas de algunos países alrededor de la IE en niños y abordan el concepto desde sus diferentes modelos, enfatizando en las investigaciones de Mayer y Salovey (1997).

En otro trabajo, Fernández y Extremera (2005) hicieron un estudio sobre la IE y la educación de las emociones desde el modelo de Mayer y Salovey. Específicamente, plantean que un desafío de la educación actual es formar no solo académicamente sino también en las emociones. $\mathrm{Al}$ respecto señalan que si bien existen programas de prevención e intervención en jóvenes que incluyen la IE, estos no tienen un sustento teórico. Por lo anterior, en su trabajo no solo explican varios programas en educación emocional, sino que además profundizan en el modelo de Mayer y Salovey, así como en los instrumentos que se han diseñado desde estos autores para medir la IE. 
También en el campo de la enseñanza, la investigación de Vásquez (2007), denominada "Inteligencia emocional en las organizaciones educativas", relaciona la IE con el ámbito educativo desde una mirada organizacional y afirma que se pretende desarrollar un nuevo campo de la psicología educativa. De acuerdo con Vásquez (2007), los impulsores de esta idea se fundamentan en las organizaciones que aprenden y crean conocimiento, de modo que centran su argumento en que es necesario que sus integrantes sean personas que aprendan y logren aprendizajes organizacionales. En este sentido, las directivas deben tener IE para poder controlar sus emociones, el estrés, la ansiedad y tener claridad para afrontar las situaciones cotidianas.

Cabe mencionar que en la literatura también se encuentran trabajos en adultos, como la investigación de Mikulic et al. (2018), quienes presentan la construcción y validación de un instrumento para evaluar la IE percibida en adultos de Buenos Aires, Argentina. Este proceso comprendió cuatro fases: (1) definición conceptual y operacional del constructo, (2) elaboración y análisis de los ítems, (3) aplicación a una muestra de 356 participantes adultos de Buenos Aires y (4) estudio de las propiedades psicométricas del inventario.

En cuanto a estudios que indaguen la relación entre la IE y el liderazgo, se puede mencionar el realizado por Martínez (2013), en el cual se destaca la importancia de la IE para afrontar los cambios permanentes que se presentan en este siglo. El autor cita a Goleman para retomar su idea de que la IE puede ser fomentada y fortalecida, y afirma que es fundamental para el desempeño personal y profesional. Posteriormente, Martínez (2013) argumenta que en el liderazgo y en las negociaciones laborales son necesarias las siguientes habilidades o componentes de la IE: autoconocimiento, autocontrol, automotivación, empatía y sociabilidad o habilidades interpersonales.

Asimismo, Sánchez et al. (2010) relacionan la IE con la autoeficacia para el liderazgo. Específicamente, indican que la IE de los líderes estudiados influyó significativamente en la autoeficacia para el liderazgo y el desempeño de los equipos, aunque, argumentan, en este último caso su influencia es parcial. Por su parte, Diez y Sánchez (2017) aseveran que las personas con mayor IE tienen una ventaja al manipular las relaciones interpersonales con su lenguaje corporal y comprender las señales emocionales de los otros. Estos autores concluyen que los factores de personalidad inciden en los estilos de liderazgo, 
así como en la IE, pero aclaran que el tipo de liderazgo puede ser anulado por factores culturales. Es decir, aunque señalan que no es fácil adaptar la IE en las organizaciones, también enfatizan que si está el deseo, esto puede traer beneficios personales y a la empresa. Al final de su reflexión, Diez y Sánchez (2017) proponen la incógnita de si las organizaciones deben formar los líderes o conseguirlos.

Otras investigaciones relacionan la IE con la calidad de vida y la convivencia. Una de ellas, realizada por Cano y Zea (2012), retoma nuevamente la obra de Daniel Goleman y propone actividades para fortalecer la inteligencia emocional y, con ella, mejorar la convivencia. Además, los autores plantean que no obstante la educación se ha centrado en desarrollar la inteligencia matemática y lingüística, las teorías de Gardner y Goleman han cambiado esa concepción, pues según Goleman hay una inteligencia emocional y una inteligencia racional.

Precisamente sobre esta distinción, la revisión empírica de Moscoso (2019) hace un estado del arte de ambos conceptos, con el fin de encontrar la mediación entre la regulación emocional y la calidad de vida de los individuos. Así, concluye que los reportes de los estudios evaluados en la integración de mindfulness con la IE evidencian beneficios significativos al bienestar de las personas. Además de este hallazgo, resalta que la IE — como constructo teórico de la psicología de la salud y la educación — está orientada al desarrollo personal del individuo, la habilidad de tomar decisiones y crear un estado de bienestar. Asimismo, Moscoso (2019) sostiene, por un lado, que la IE es un modelo conceptual de habilidades que pueden ser adquiridas y mejoradas a través del entrenamiento de carácter cognitivo-emocional, y, por otro, que las habilidades que la representan están compuestas por los siguientes elementos: percepción de los estados emocionales, la comprensión de la naturaleza afectiva y la habilidad de manejar el proceso de regulación emocional con el fin de llegar a un estado de bienestar y desarrollo personal.

Igualmente, en las memorias del II Congreso Internacional de Inteligencia Emocional (Fernández et al., 2011) se ahonda en el concepto de IE, pero desde las diferentes pruebas o formas de medida, y se citan varios trabajos investigativos. En este documento también se asocian diferentes categorías o dimensiones de lo humano (maltrato físico, optimismo, ansiedad, memoria 
autobiográfica, estilos de vida, afrontamiento, apego, conductas suicidas, depresión, psicopatología, etc.). Finalmente, se exponen las investigaciones en el campo educativo, se abordan temas desde la empatía, las habilidades sociales, el bullying, el afrontamiento y el humor, hasta programas de formación en competencias socioemocionales. Por último, las memorias describen diversas experiencias educativas.

En contraste con los estudios referenciados, cabe señalar que también hay investigadores que ponen en duda el sustento científico del concepto de IE. Este es el caso de Manrique (2015), quien cuestiona el carácter científico de la teoría de Daniel Goleman, creador del concepto. Concretamente, Manrique (2015) plantea que se volvió un término de moda y que el concepto se basa en las ideas rogerianas y del pensamiento budista. Por otro lado, argumenta que no se ahonda teóricamente en los conceptos de emociones y de inteligencia, sino que son tratados de una forma muy coloquial. Igualmente, el autor considera que el concepto de IE se enfoca en que los sujetos construyan una mejor versión de sí mismos y en que los traumas se deben a factores externos como la familia, el entorno, la crianza, etc., con lo cual se trata al sujeto como víctima. Dice además que quienes trabajan la IE plantean que esta se puede entrenar, lo que para él causa problemas debido a la contención emocional, de allí que haga una comparación con la teoría psicoanalítica de Freud al producir comportamientos neuróticos. En la última parte de su reflexión, Manrique (2015) señala que la inteligencia emocional busca que todos seamos iguales y que solo expresemos las emociones positivas o deseables en la convivencia, lo cual resulta imposible para el autor, ya que en los seres humanos se mueven deseos y pasiones que hacen difícil predecir los comportamientos.

Finalmente, se debe destacar que las investigaciones coinciden al citar los diferentes modelos y autores representativos de la IE, especialmente a Mayer y Salovey, por una parte, y a Goleman, por otra. También resaltan la importancia de la IE en el desarrollo personal, académico y profesional de los individuos, al tiempo que algunos autores relacionan la IE con las habilidades del liderazgo, la calidad de vida y la convivencia. Como se pudo evidenciar, solo se encontró una investigación que refuta el sustento teórico y científico del concepto de IE. 


\section{Inteligencia emocional en entornos universitarios}

Se encontraron varias investigaciones respecto a la IE en entornos universitarios. Una de ellas es la realizada por Rocío Fragoso (2018b), en la cual plantea la importancia del desarrollo socioemocional en los estudiantes universitarios, dado que son habilidades que se requieren para el buen desempeño profesional.

Aunque reconoce que no existen muchas estrategias para desarrollarlas, Fragoso (2018b) sintetiza varios trabajos en que se proponen algunas: por una parte, presenta el estudio de Landau y Meirovich (2011), quienes correlacionan la creación de ambientes participativos de aprendizaje en el salón de clases con el desarrollo de la IE. Por otra, cita la sistematización que hace Mortiboys (2016) de una serie de prácticas y técnicas que pueden ser desarrolladas fácilmente en las aulas de educación superior para fomentar la IE, aunque señala que no tienen una base teórica definida. Finalmente, se refiere al programa de aprendizaje socioemocional Ruler, un acrónimo de las habilidades que se espera desarrollar: recognizing (reconocer las diferentes manifestaciones de los estados emocionales propios o ajenos), understanding (comprender las causas y consecuencias de las emociones), labeling (etiquetar emociones básicas y complejas), expressing (expresar emociones de manera constructiva) y regulating (regular las emociones de forma adecuada).

Además, Fragoso (2018b) plantea cinco desafíos que enfrenta la educación superior para desarrollar las habilidades socioemocionales: (1) inclusión de la IE en el currículum universitario, (2) la integración de la organización educativa en el desarrollo de la IE, (3) la inclusión de los directivos en el desarrollo de la IE, (4) la sensibilización y capacitación de los docentes universitarios para la promoción de la IE en el aula, y (5) el compromiso de los estudiantes hacia el desarrollo de la IE dentro del aula.

Posteriormente, la autora ahonda en el modelo de IE de Mayer y Salovey como base de la propuesta que propone. Específicamente, su modelo plantea cuatro aspectos, cada uno con sus respectivas estrategias: (1) en el desarrollo de la percepción y la expresión emocional sugiere hacer una lista emocional y análisis de fotografías, documentales o películas; (2) en el desarrollo de la facilitación emocional propone fomentar ambientes emocionalmente seguros y ambientes de aprendizajes participativos. En cuanto al (3) desarrollo de la comprensión 
de emocional, propone la secuencia interrumpida y juego de roles, y (4) en el desarrollo de la regulación emocional plantea hacer simulaciones y técnicas de relajación.

Por su parte, Niño et al. (2017) argumentan que es indispensable determinar la importancia que las instituciones universitarias dan a la IE en el éxito profesional y personal de sus estudiantes. Con esto en mente, los autores realizaron una investigación inductivo-deductiva basada en estudios de casos para plantear una nueva teoría. Específicamente, concluyen que es necesario un cambio cultural en las instituciones universitarias para incluir la educación emocional, para lo cual se debe entrenar a los docentes en un modelo de relaciones cooperativas. Así, argumentan que es importante contemplar un enfoque que fomente la IE desde la educación primaria hasta la universitaria.

También se debe mencionar la tesis doctoral de la autora Pozo (2016), quien describe un programa de competencia emocional en educación superior realizado en un entorno virtual de aprendizaje y desde un enfoque transcultural. De acuerdo con la investigadora, los resultados mostraron la eficacia del programa, por lo tanto propone alinearlo a los planes de estudio de las entidades participantes.

Por su parte, Fragoso (2015) argumenta que los niños y adultos deben tener unas competencias específicas para que sean exitosos y responsables, las cuales están relacionadas con la IE. En cuanto a la educación superior, la autora se refiere a las habilidades y competencias del Proyecto Tuning, el cual plantea que cualquier profesional debe trabajar en equipo, tener habilidades interpersonales, trabajar por metas comunes, tomar buenas decisiones, entre otras. Luego de hacer un recorrido histórico para mostrar la evolución del concepto de IE desde 1900, cuando comenzó el enfoque psicométrico de la inteligencia, hasta la teoría reformulada de Mayer y Salovey, Fragoso (2015) aborda el concepto de competencia emocional desde el modelo de Carolyn Saarni y el trabajo de Rafael Bisquerra, quien define las competencias emocionales como

el conjunto de conocimientos, capacidades, habilidades y actitudes necesarios para tomar conciencia, comprender, expresar y regular de forma apropiada los fenómenos emocionales cuya finalidad es aportar un valor añadido a las funciones profesionales y promover el bienestar personal y social. (Fragoso, 2015, p. 120) 
Concretamente, este modelo se compone de cinco elementos: conciencia emocional, regulación emocional, autonomía emocional, competencia social, competencias para la vida y el bienestar.

Desde otra perspectiva de análisis, Páez y Castaño (2015) no encontraron diferencias significativas de Ie por género, pero sí por carrera, y evidenciaron una correlación entre la IE y el rendimiento académico. Ariza (2017), por su parte, describe cómo influye la IE y el afecto entre docentes y estudiantes de educación superior en su rendimiento. De esta manera, concluye que existe una relación significativa entre el rendimiento académico y la IE, pues esta contribuye a la capacidad de resolver problemas, trabajar con los demás de forma colaborativa, lograr metas y propósitos en la vida. El trabajo de Ariza (2017) evidencia que los docentes que gestionan ambientes de aprendizaje y estrategias pedagógicas generan emociones positivas que motivan y potencian la inteligencia emocional que incide en el éxito académico. Finalmente, resalta que para hacer esto es necesario implementar programas de educación emocional como parte del currículo.

En cuanto a las investigaciones sobre la medición de la inteligencia emocional, el género y el tipo de carrera de los estudiantes de educación superior, se puede mencionar el estudio de Rodríguez et al. (2019), quienes analizaron el nivel de la IE según el género. Específicamente, los autores encontraron que mientras las mujeres tienen los mayores niveles de atención y autocomprensión, los hombres registran mayor puntuación en comprensión y reparación.

En el campo de los estudios universitarios, la investigación de Salas y García (2010) establece un perfil de habilidades de inteligencia emocional por carreras, para lo cual evaluaron las dimensiones de atención, claridad y reparación. La dimensión atención evalúa las percepciones y pensamientos que tienen los estudiantes acerca de sus emociones. La dimensión reparación indica que los participantes se orientan al mantenimiento de los estados de ánimo agradables o a modificar los desagradables. La dimensión claridad indaga por la comprensión del propio estado de ánimo.

De acuerdo con el estudio, estas dimensiones muestran un patrón común, con el siguiente orden de puntajes: reparación, atención y claridad para las carreras de ingeniería de producción, ingeniería eléctrica y urbanismo, 
lo cual indica que mantienen sus estados emocionales agradables o modifican los desagradables, y reconocen sus emociones, pero no las comprenden. En el caso de arquitectura, Salas y García (2010) explican que esta se diferencia de las otras carreras porque tiene una configuración distinta de las dimensiones: en primer lugar estaría la dimensión de la atención, luego la reparación y por último la claridad.

Por su parte, Fragoso (2018a) realizó una investigación sobre la IE en estudiantes de educación superior de diferentes programas. Como parte del estudio, la investigadora plantea que la mayoría de las evaluaciones de la Ie se realiza de manera cuantitativa, sin incluir la experiencia personal de los individuos. En cuanto a los resultados, Fragoso (2018a) encontró diferencias en la IE entre los estudiantes de diferentes carreras: se destacan los estudiantes de psicología con atención, claridad y reparación emocional. Los estudiantes de lingüística y literatura hispana por su expresión emocional. Finalmente, concluye que los estudiantes con altos puntajes en el TMMs-24 tienden a describir mayor número de ejemplos que desarrollan las habilidades de la IE según el modelo de Mayer y Salovey.

En esta misma línea, Gilar et al. (2018) implementaron un Programa de Capacitación Avanzada en Competencia Emocional (Aтрес) con el fin de mejorar las habilidades emocionales en estudiantes de educación superior, aumentar la capacidad de trabajar en grupos para resolver casos complicados y potenciar la capacidad de aprender de forma autónoma. Los investigadores concluyen que la posibilidad de trabajar en grupos para resolver casos aplicados y la capacidad de aprender de forma autónoma (plataforma e-learning) convierten al programa en un instrumento novedoso y eficaz para mejorar la competencia emocional.

Asimismo, se encontraron investigaciones que le dan gran importancia a la IE en la formación universitaria, pues plantean que es fundamental para el desempeño profesional futuro de los estudiantes. Algunas relacionan la IE con los lineamientos de entidades internacionales como la UnEsco, la Ocde y el Proyecto Tunning. Otros estudios, como se mencionó, profundizan en los niveles de la IE y su relación con el género y el tipo de carreras. Por último, se encuentran algunos trabajos que citan o proponen programas para desarrollar la inteligencia emocional en estudiantes universitarios. 
Complementando lo anterior, también se identificaron investigaciones que relacionan la IE con un desempeño profesional idóneo. Al respecto, Duque (2012) asevera que las habilidades cognitivas no son suficientes para un buen desempeño profesional de los individuos en las organizaciones, sino que también son fundamentales las emociones y la IE. Al inicio de su trabajo, el autor define el concepto de emociones y rasgos emocionales, y aborda la diferencia entre emociones positivas y negativas. Luego, profundiza en la interferencia de las emociones en el desempeño profesional y en las relaciones laborales, diferenciando las formas de comportamiento desde el coeficiente cognitivo y el coeficiente emocional, especialmente en roles gerenciales.

De acuerdo con su análisis, los directores, los ejecutivos o las personas con cargos superiores deben mostrar altos niveles de empatía, autocontrol de las emociones y serenidad. Con base en las reflexiones de Goleman sobre los entornos laborales, Duque (2012) describe la IE como la capacidad de gestionar las emociones propias y utilizarlas, en el caso de las empresas, en función de un mejor desempeño y rendimiento efectivo en el puesto de trabajo. Igualmente, hace un recorrido histórico del concepto desde diferentes autores y finaliza resaltando su importancia en los roles de liderazgo, la eficiencia y la eficacia en el trabajo, la salud en el entorno laboral, etc.

Asimismo, Duque et al. (2017) analizan la incidencia de la IE sobre las competencias laborales y concluyen que esta incide en las competencias laborales, ya que reconocer las emociones, la comunicación, la capacidad de negociación, el trabajo en equipo, entre otras habilidades, favorece un mejor desempeño laboral. Igualmente, la investigación de Carmona et al. (2015) concluye que, efectivamente, la IE incide de manera positiva en el desempeño laboral.

Finalmente, las investigaciones que relacionan la IE con el desempeño laboral coinciden en sus conclusiones, ya que el manejo de las emociones, la empatía, las habilidades de negociación, el trabajo en equipo y la asertividad contribuyen a cumplir las funciones encomendadas y generar un buen clima laboral. Por lo anterior, es indispensable que se incluya la IE en los diferentes currículos de las carreras y se desarrolle y fortalezca en la formación de los profesionales civiles y militares, con el fin de tener una formación integral que articule el conocimiento disciplinar y la formación humana. Así, estos estudios 
demuestran que el desempeño de un profesional no depende solamente de los conocimientos de su disciplina, sino también de la relación que establezca con los demás.

\section{Inteligencia emocional en entornos militares}

$\mathrm{Al}$ indagar la IE en entornos militares se encontraron algunas investigaciones, una de ellas realizada en Venezuela por Labarca y Estaba (2010), la cual tuvo como objetivo analizar la inteligencia emocional como herramienta gerencial que permita mejorar el proceso de toma de decisiones gerenciales de los directores y jefes de dependencias del Ejército Nacional Bolivariano. Los autores consideran que el personal no solo debe tener conocimiento y habilidades para ejercer su profesión, sino que además es muy importante la inteligencia emocional para dirimir conflictos, trabajar en equipo, generar confianza, tener un liderazgo positivo y tomar decisiones acertadas. Los resultados señalan que el $75 \%$ de los directores y jefes de dependencias del comando general del Ejército Nacional Bolivariano poseen un muy buen nivel de inteligencia emocional, lo cual indica que reconocen sus emociones y la de los demás, pueden automotivarse y tomar decisiones acertadas para la ejecución de actividades administrativas y operacionales.

Por su parte, Gerald Sewell (2011) destaca el papel del líder en el Ejército, lo que debe ser, saber y hacer. Resalta la capacidad de resiliencia del líder:

El líder resiliente rápidamente puede recuperarse de los contratiempos, la conmoción, las lesiones, las adversidades y el estrés, mientras mantiene el enfoque de la misión y de la organización. Su resiliencia descansa en la voluntad, el impulso interno que lo motiva a seguir adelante, incluso cuando se siente agotado, hambriento, temeroso, con frío y mojado. (p. 2)

Así, plantea que la resiliencia no se debe desarrollar solo en los líderes de combate, sino en todos los integrantes del Ejército, por eso la Escuela de Comando y Estado Mayor del Ejército de los Estados Unidos, junto con la Universidad de Pensilvania, ha creado el Programa de Acondicionamiento Integral del Soldado, que trabaja cinco aspectos para inculcar la resiliencia: físico, emocional, espiritual, familia y social. De esta manera, relaciona la inteligencia emocional con la capacidad de resiliencia y plantea que si una persona 
es capaz de comprender sus emociones y las de los demás, podrá tener mayor éxito, pues la persona equilibrada emocionalmente podrá afrontar la adversidad. Específicamente, Sewell (2011) se basa en el modelo de Bar-On con los dominios intrapersonal, interpersonal, adaptabilidad, control de estrés y estado de ánimo general, con los cuales propone evaluar el cociente emocional. Finalmente, enfatiza la necesidad de trabajar la inteligencia emocional para el desarrollo de la resiliencia en los militares.

En relación con la investigación anterior, se encontró un programa sobre la resiliencia en contextos militares desarrollado por García y Bardera (2013). Las autoras señalan que si bien en el siglo XXI el peso de la preparación militar se ha centrado en desarrollar aspectos físicos, técnicos y tácticos, ahora se hace necesario la preparación o el entrenamiento psicológico. Cabe señalar que aunque las variables psicológicas son muchas, García y Bardera (2013) trabajan en la resiliencia, ya que esta permite reducir los riesgos psicosociales de la profesión militar. En este sentido, entienden la resiliencia como la suma de las siguientes variables: individuales (como la personalidad, la inteligencia emocional, las estrategias de afrontamiento, etc.), psicosociales (donde se incluye el apoyo familiar) y organizacionales o de unidad (como la cultura o el clima organizacional, donde se integra el liderazgo o el apoyo de unidad). Luego de esta descripción, presentan su propuesta de entrenamiento, la cual incluye fases de intervención, actividades y objetivos de las actividades a nivel individual, grupal y familiar. Finalmente, exponen los programas y contenidos que en su consideración se deben desarrollar en el personal militar.

Otra investigación que se deben mencionar es la que realizaron Cutipa y Ortega (2016), en la cual correlacionaron la IE con los estilos de liderazgo y cuyos resultados muestran una relación significativa entre la IE y el estilo de liderazgo transformacional. Igualmente, se revisó una compilación de monografías de las Fuerzas Armadas Españolas (Ministerio de Defensa Nacional, 2013), las cuales relacionan el liderazgo con las emociones o la IE. Asimismo, estos trabajos desarrollan el concepto de liderazgo, los estilos de liderazgo, las características del líder, el liderazgo militar, entre otros aspectos, y abordan conceptos de competencias e inteligencia emocionales, así como la IE en la formación militar.

En este tema específico, Tappen (2007) contextualiza el mando, el liderazgo y señala que la IE no debe descartar los conocimientos técnicos y profesio- 
nales, sino centrar ciertas cualidades personales como la iniciativa, la empatía, la adaptabilidad y la persuasión, entre otras. El autor cita a Daniel Goleman para resaltar que las condiciones intelectuales no son garantía de éxito en el ámbito profesional, sino tan solo uno de los factores, y a partir de esta consideración define la IE como "la capacidad para reconocer sentimientos en sí mismo y en los demás, siendo hábil para manejarlos al trabajar con ellos”. Tappen (2007) continúa señalando que se debe poder identificar y manejar los sentimientos, así como expresarlos adecuadamente y con efectividad, como lo define Platón: "Cuidado e inteligencia para conducir la propia vida; un equilibrio y una sabiduría templados". Así, luego de describir las diferentes aptitudes personales: de autorregulación, motivación y habilidades sociales, que se resumen en el autocontrol, el autor cierra su investigación con esta reflexión: no se puede ser conductor si no se tiene la capacidad de conducirse uno mismo, su propio actuar, y ese liderazgo solo se logra desarrollando las características definidas del liderazgo interpersonal. En este sentido, quienes no alcanzan a descubrir e interiorizar la IE y el liderazgo personal, no van a identificar sus errores, de allí que tener visión, creatividad, paciencia y perseverancia desempeña un papel importante en el ejercicio del mando con IE, pues "la excelencia depende más de las competencias 'emocionales' que de las cognitivas'.

Pedraza (2013), por su parte, hace un análisis de los conceptos de liderazgo y liderazgo militar involucrando la IE que usan los militares. Cita a Goleman, padre de la IE, para plantear que un liderazgo ejemplar requiere de la adecuada combinación entre "el corazón y la cabeza", entre el sentimiento y el pensamiento, aspectos esenciales que los líderes militares deben aplicar en la institución. La autora define el liderazgo emocional como la capacidad que se tiene para manejar las emociones de forma positiva y afectiva para liderar con eficacia. Después de estudiar a los diferentes líderes militares de la historia, afirma que todos los integrantes de las Fuerzas Militares deben explotar las aptitudes emocionales con el propósito de influir en todos sus subordinados y desarrollar el autocontrol para que tengan visión, creatividad, paciencia y perseverancia, cualidades necesarias para desarrollar la IE. Como conclusión, Pedraza (2013) recomienda que la doctrina se debe desarrollar con la premisa de un modelo de liderazgo emocional que tenga en cuenta las características, las cualidades, las habilidades y el autocontrol de los líderes. 
Por otro lado, Gossweiler (2017) concluye que la IE no es un proceso separado de la toma de decisiones y el proceso de formación de los cadetes de la Escuela Naval Militar de Bolivia. Su investigación muestra que no se evidencia un nivel de IE antes, durante y finalizando la formación militar, por lo cual plantea que las instituciones militares deberían reforzarla y enfocarse en una formación integral. En este mismo campo, Solís (2014) elaboró un manual de técnicas de control emocional para mejorar el trabajo en equipo y permitir al personal militar obtener una adecuada respuesta al expresar sus emociones y resolver sus conflictos personales para después alcanzar un trabajo en grupo equilibrado y mantener la empatía de sus integrantes. En este sentido, el trabajo de Solis (2014) se ubica en el paradigma crítico-propositivo con el objetivo de plantear alternativas al problema del control emocional y el trabajo en equipo.

Esta revisión de las investigaciones sobre la IE en entornos militares evidencia que la mayoría la asocian con el liderazgo y la toma de decisiones, ya que estas dos capacidades son fundamentales en el personal militar, especialmente en oficiales. Así, se plantea que es indispensable desarrollar y fortalecer la IE en los currículos de la formación militar, ya que los militares deben aprender a manejar sus emociones, ser asertivos al dar las órdenes o retroalimentar a sus subalternos, trabajar en equipo, comprender a los demás, autocontrolarse, etc. Finalmente, cabe seńalar que algunas investigaciones destacan la resiliencia como otra capacidad que se debe trabajar en los estudiantes militares, capacidad relacionada con la IE.

\section{Inteligencia emocional y personalidad}

Por último, se analizan las investigaciones que relacionan la IE con la personalidad, pues las dos variables son interdependientes y se deben tener en cuenta en los procesos educativos. En Colombia, Contreras et al. (2010) realizaron un estudio denominado "Personalidad, inteligencia emocional y afectividad en estudiantes universitarios de áreas empresariales: implicaciones para la formación de líderes". Los resultados muestran niveles altos de neuroticismo y bajos de extroversión, apertura a la experiencia y amabilidad, los cuales se incrementan durante el proceso formativo; niveles intermedios de inteligencia emocional y afectividad positiva predominante. Al respecto, Morales (2004) se plantea la pregunta de si la inteligencia es un rasgo más de la personalidad, 
para lo cual analiza varias teorías y propone que es necesario tener en cuenta los aspectos culturales y las diferencias individuales. Finalmente, cita a Stemberg, Gardner y la teoría de sistemas que relaciona la inteligencia y la personalidad.

Otra investigación que relaciona la personalidad con la IE fue realizada por Bernarás et al. (2011), quienes plantean que existen pocas investigaciones en la edad adulta y en la vejez sobre IE, pues la mayoría se centran en niños, niñas y adolescentes. Por lo anterior, hacen una revisión de las investigaciones que indagan la IE en jóvenes, adultos y adultos mayores en relación con otras categorías (autoconcepto, factores de la personalidad, ansiedad, creatividad, etc.) y diferenciando por género.

Espinosa (2011), por su parte, estudia el carácter, el destino y las emociones desde una perspectiva filosófica y neurobiológica. Define el carácter como el crisol de las emociones y ve al ser humano desde una concepción biocultural, en donde los genes y la socialización inciden en el carácter y el destino. Afirma que las emociones son el puente entre los dos, ya que es la que informa, valora y motiva las acciones del sujeto. Luego relaciona la ética, haciendo una reflexión entre el ser y el deber ser, entre el logos y el pathos, para lo cual cita varios filósofos. Pasa posteriormente a la discusión científica y biológica de las emociones, a las cuales relaciona con la socialización y la cultura.

Finalmente, el trabajo de Pérez et al. (2009) muestra correlaciones significativas entre las variables que analizaron: el optimismo se relaciona con la empatía, la reparación emocional, la resolución positiva de los problemas, entre otras. Por último, se encontró la investigación de Rodríguez (2020), quien demuestra que los rasgos de la personalidad guardan relación directa y positiva con la inteligencia emocional.

En síntesis, las investigaciones que relacionan factores de la personalidad con la IE encuentran correlaciones significativas con el optimismo, la extroversión, la afectividad positiva, la amabilidad, la responsabilidad, entre otros aspectos.

\section{Conclusiones}

La revisión de las investigaciones sobre IE encontradas y analizadas permiten concluir lo siguiente: (1) Las investigaciones realizan un recorrido por los diferentes modelos y concepciones de la IE y la mayoría se basan en las 
propuestas de Mayer y Salovey y de Goleman. (2) Se resalta la importancia de la IE en el desarrollo personal, académico y profesional de los individuos. (3) Se relaciona la IE con las variables de liderazgo, calidad de vida, convivencia y personalidad. (4) Se resalta la importancia de la IE en la formación universitaria, pues será indispensable para el futuro quehacer profesional. (5) La IE es fundamental en el entorno laboral, ya que el manejo de las emociones, la empatía, las habilidades de negociación, el trabajo en equipo y la asertividad contribuyen a cumplir las funciones encomendadas y generar un buen clima laboral. (6) La IE en los entornos militares ha tomado gran importancia, pues se asocia con las capacidades de liderazgo, toma de decisiones y resiliencia, capacidades indispensables en el desempeño de los militares. Por tanto, se plantea incluir el desarrollo y fortalecimiento de la IE en los programas de formación militar.

\section{Referencias}

Antunes, C. (2006). Inteligencias múltiples: cómo estimularlas y desarrollarlas. Alfaomega y Nenarcea.

Ariza, M. (2017). Influencia de la inteligencia emocional y los afectos en la relación maestro-alumno, en el rendimiento académico de estudiantes de educación superior. Educación y Educadores, 20(2), 193-210. https://doi.org/10.5294/edu.2017.20.2.2

Bernarás, E., Garaigordobil, M., \& De las Cuevas., C. (2011). Inteligencia emocional y rasgos de personalidad. Influencia de la edad y el género durante la edad adulta y la vejez. Boletín de Psicología, O(103), 75-88. https://www.uv.es/seoane/boletin/previos/N103-5.pdf

Bisquerra, R., \& Pérez, N. (2007). Las competencias emocionales. Educación XX1, (10), 61-82. https://doi.org/10.5944/educxx1.1.10.297

Bisquerra, R., Pérez, J., \& García, E. (2015). Inteligencia emocional en educación. Editorial Síntesis. https://www.sintesis.com/data/indices/9788490770788.pdf

Bolaños, A., Bolaños, E., Gómez, D., \& Escobar, A. (2013). Origen del concepto inteligencia emocional [Tesis de maestría, Universidad de San Carlos de Guatemala]. Repositorio institucional. http://www.repositorio.usac.edu.gt/8601/1/19\%20MDU\%20T-30742494-UrzuaNavas.pdf

Cano, S., \& Zea, M. (2012). Manejar las emociones, factor importante en el mejoramiento de la calidad de vida. Revista Logos, Ciencia \& Tecnología, 4(1), 58-67. https://doi. org/10.22335/rlct.v4i1.169

Carmona, P., Vargas, J., \& Rosas, R. (2015). Influencia de la inteligencia emocional en el desempeño laboral. Sapienza Organizacional, 2(3), 53-68. http://dx.doi.org/10.14482/ psdc.35.1.11155 
Contreras, F., Barbosa, D., \& Espinosa, J. (2010). Personalidad, inteligencia emocional y afectividad en estudiantes universitarios de áreas empresariales: implicaciones para la formación de líderes. Perspectivas en Psicología, 6(1), 65-79. https://doi.org/10.15332/s17949998.2010.0001.05

Cutipa, L., \& Ortega, A. (2016). Inteligencia emocional y liderazgo en oficiales de armas con mando de tropa de la tercera división militar del Ejército, Guarnición Arequipa [Tesis de pregrado, Universidad Nacional de San Agustín de Arequipa]. Repositorio institucional. http:// repositorio.unsa.edu.pe/handle/UNSA/3511?show=full

Danvila, I., \& Sastre, M. (2010). Inteligencia emocional: una revisión del concepto y líneas de investigación. Cuadernos de Estudios Empresariales, (20), 107-126. https://dialnet. unirioja.es/servlet/articulo? codigo $=3934705$

Diez, S., \& Sánchez, E. (2017). Influencia de la personalidad y la inteligencia emocional en los estilos de liderazgo. Revista Empresarial, 11(43), 22-28. https://dialnet.unirioja.es/ servlet/articulo? codigo $=6185663$

Dueñas, M. (2002). Importancia de la inteligencia emocional: un nuevo reto para la orientación educativa. Educación XX1, (5), 77-96. https://doi.org/10.5944/educxx1.5.1.384

Duque, J. (2012). Emociones e inteligencia emocional: una aproximación a su pertinencia y surgimiento en las organizaciones. Libre Empresa, 9(2), 147-169. https://revistas. unilibre.edu.co/index.php/libreempresa/article/view/2979

Duque, J., García, M., \& Hurtado, A. (2017). Influencia de la inteligencia emocional sobre las competencias laborales: un estudio empírico con empelados del nivel administrativo. Estudios Gerenciales, 33(144), 250-260. https://doi.org/10.1016/j.estger.2017.06.005

Espinosa, L. (2011). Carácter, destino y emociones. Logos. Anales del Seminario de Metafísica, (44), 303-329. http://dx.doi.org/10.5209/rev_asEM.2011.v44.36889

Extremera, N., \& Fernández, P. (2004). El papel de la inteligencia en el alumnado: evidencias empíricas. Revista Electrónica de Investigación Educativa, 6(2), 2-17. http://www. scielo.org.mx/scielo.php?script=sci_arttext\&pid=\$1607-40412004000200005

Fernández, M. (2013). La inteligencia emocional. Revista de Clases Historias, (377), 1-12.

Fernández, P., \& Extremera, N. (2005). La inteligencia emocional y la educación de las emociones desde el modelo de Mayer y Salovey. Revista Interuniversitaria de Formación del Profesorado, 19(3), 63-93. https://www.redalyc.org/pdf/274/27411927005.pdf

Fernández, P., \& Ruíz, D. (2008). La inteligencia emocional en la educación. Revista Electrónica de Investigación Psicoeducativa, 15(6), 421-436. http://www.investigacion-psicopedagogica.org/revista/articulos/15/espannol/Art_15_256.pdf

Fragoso, R. (2015). Inteligencia emocional y competencias emocionales en educación superior, ¿un mismo concepto? Revista Iberoamericana de Educación Superior, 6(16), 110-125. https://doi.org/10.1016/j.rides.2015.02.001

Fragoso, R. (2018a). Inteligencia emocional en estudiantes de educación superior. Análisis a través de técnicas mixtas. International Journal of Developmental and Educational Psychology, 1(2), 231-239. https://doi.org/10.17060/ijodaep.2018.n2.v1.1334 
Fragoso, R. (2018b). Retos y herramientas generales para el desarrollo de la inteligencia emocional en las aulas universitarias. Praxis Educativa, 22(3), 47-55. http://dx.doi.org/10.19137/ praxiseducativa-2018-220305

García, M., \& Bardera, M. (2013). Programación de la preparación psicológica. Revista Ejército, (863), 42-48. https://ejercito.defensa.gob.es/Galerias/multimedia/revista-ejercito/2013/R_Ejercito_863.pdf

García, M., \& Giménez-Mas, S. (2010). La inteligencia emocional y sus principales modelos: propuesta de un modelo integrador. Espiral. Cuadernos del Profesorado, 3(6), 43-52. https://dialnet.unirioja.es/servlet/articulo?codigo=3736408

Gilar-Corbi, R., Pozo-Rico, T., \& Castejón-Costa, J. (2019). Desarrollando la inteligencia emocional en educación superior: evaluación de la efectividad de un programa en tres países. Educación XX1, 22(1), 161-187. https://doi.org/10.5944/educxx1.19880

Goleman, D. (1996). La inteligencia emocional. Vergara.

Gossweiler, L. (2017). Inteligencia emocional y toma de decisiones de mando en cadetes de la Escuela Naval Militar [Tesis de pregrado, Universidad Mayor de San Andrés]. Repositorio institucional. https://repositorio.umsa.bo/handle/123456789/10991

Labarca, F., \& Estaba, R. (2010). Inteligencia emocional como herramienta gerencial para la toma de decisiones de los directores del comando general del Ejército Nacional Bolivariano. Revista del Centro de Investigación de Ciencias Administrativas y Gerencias, 7(2), 63-80. https://dialnet.unirioja.es/servlet/articulo? codigo=3706285

Manrique, R. (2015). La cuestión de la inteligencia emocional. Revista Asociación Española de Neuropsiquiatría, 35(128), 801-814. http://dx.doi.org/10.4321/S021157352015000400008

Martínez, A. (2013). La inteligencia emocional: una herramienta necesaria para el liderazgo. Concepto y componentes. Revista de Investigaciones UNAD, 12(1), 149-158. https:// doi.org/10.22490/25391887.1164

Mikulic, I., Crespi, M., \& Caballero, R. (2018). Construcción de un inventario de inteligencia emocional percibida para adultos. Ciencias Psicológicas, 12(1), 110-121. http://dx.doi. org/10.22235/cp.v12i1.1602

Ministerio de Defensa Nacional. (2013). Monografías 136. El liderazgo de las Fuerzas Armadas del siglo XXI. Escuela Superior de las Fuerzas Armadas. Ministerio de Defensa. https://publicaciones.defensa.gob.es/media/downloadable/files/links/m/o/ monografia_136.pdf

Moscoso, M. (2019). Hacia una integración de mindfulness e inteligencia emocional en psicología y educación. Liberabit: Revista Peruana de Psicología, 25(1), 107-117. https://doi. org/10.24265/liberabit.2019.v25n1.09

Niño, J., García, E., \& Caldevilla, D. (2017). Educación emocional y educación universitaria: una aproximación. Revista de Comunicación de la SEECI, (43), 15-27. https://doi. org/10.15198/seeci.2017.43.15-27 
Páez, M., \& Castaño, J. (2015). Inteligencia emocional y rendimiento académico en estudiantes universitarios. Psicología desde el Caribe, 32(2), 268-285. http://dx.doi.org/10.14482/ psdc.32.2.5798

Pedraza, V. (2013). Liderazgo emocional al interior de las Fuerzas Militares [Tesis de especialización, Universidad Militar Nueva Granada]. Repositorio institucional. https://repository. unimilitar.edu.co/bitstream/handle/10654/10882/ENSAYO\%20LIDERAZGO\%20 EMOCIONAL\%20\%20FFFMM.pdf;jsessionid=B01E01BA09629A417A35C90D89E56ECE? sequence $=2$

Pérez,N.,Cantero,M., \&Castejón,J. (2009).¿Contribuyeeloptimismoenlainteligenciaemocional, la empatía y la personalidad? International Journal of Developmental and Educational Psychology, 4(1), 33-43. https://www.redalyc.org/pdf/3498/349832323003.pdf

Pozo, T. (2016). La inteligencia emocional como clave para el desarrollo de competencias en la educación superior: un enfoque transcultural [Tesis doctoral, Universidad de Alicante]. Repositorio institucional. https://dialnet.unirioja.es/servlet/tesis? codigo $=121462$

Rodríguez, F. (2020). Rasgos de personalidad e inteligencia emocional del personal de seguridad pública de Huancayo. Revista Integración Académica en Psicología, 8(2), 106-114. https://integracion-academica.org/anteriores/21-volumen-4-numero-12-2016/145-rasgos-de-personalidad-e-inteligencia-emocional-del-personal-de-seguridad-publica-de-huancayo

Rodríguez, J., Sánchez, R., Ochoa, L., Cruz, I., \& Fonseca, R. (2019). Niveles de inteligencia emocional según género de estudiantes en la educación superior. Espacios, 40(31), 1-11. https://www.revistaespacios.com/a19v40n31/a19v40n31p26.pdf

Salas, A., \& García, R. (2010). Perfil de inteligencia emocional y carreras universitarias en estudiantes de la Universidad Simón Bolívar. Revista de Ciencias Sociales, 16(2), 226-238. http://ve.scielo.org/scielo.php?script=sci_arttext\&pid=S1315-95182010000200004\&ln$\mathrm{g}=\mathrm{es} \& \mathrm{nrm}=\mathrm{iso} \& \mathrm{t} \operatorname{lng}=\mathrm{es}$

Sánchez, J., Caballo, T., Machín, E., Gutiérrez, A., \& Quintana, R. (2010). Influencia de la inteligencia emocional en la autoeficacia para el liderazgo. International Journal of Developmental and Educational Psychology, 1(1), 43-50. https://www.redalyc.org/ pdf/3498/349832324004.pdf

Sewell, G. (2011). Cómo la inteligencia emocional puede influir positivamente. Military Review, O(4), 1-7. https://www.armyupress.army.mil/Portals/7/military-review/Archives/ Spanish/MilitaryReview_20110831_art004SPA.pdf

Solís, D. (2014). El control emocional y el trabajo en equipo en los aspirantes a soldados de arma y servicio de los paralelos A, B, C y D promoción 2013-2015 de la Escuela de Formación de Soldados del Ejército Ecuatoriano "Vencedores del Cenepa" de la Ciudad de Ambato, Provincia de Tungurahua [Tesis de pregrado, Universidad Técnica de Ambato]. Repositorio institucional https://repositorio.uta.edu.ec/ handle/123456789/9306

Tappen, G. (2007). Liderazgo e inteligencia emocional. Revismar, 2, 156-162. https://www. yumpu.com/es/document/read/25732891/liderazgo-e-inteligencia-emocional-revista-de-marina 
Trujillo, M., \& Rivas, L. (2005). Orígenes, evolución y modelos de inteligencia emocional. INNOVAR Revista de Ciencias Administrativas y Sociales, 15(25), 9-24. https:// www.redalyc.org/pdf/818/81802502.pdf

Vásquez, F. (2007). Inteligencia emocional en las organizaciones educativas. Psicogente, 10(17), 42-59. http://revistas.unisimon.edu.co/index.php/psicogente/article/view/1573

\section{Bibliografía consultada}

Aguaded, M. (2017). Estrategias para potenciar la inteligencia emocional en educación infantil: aplicación del modelo de Mayer y Salovey. Tendencias Pedagógicas, 30, 175-190. https:// doi.org/10.15366/tp2017.30.010

Fernández, P., Extremera, N., Palomera, R., Ruíz, D., Salguero, J., \& Cabello. R. (2011). Inteligencia emocional: 20 años de investigación y desarrollo. Fundación Botín. https:// www.fundacionbotin.org/paginas-interiores-de-una-publicacion-de-la-fundacion-botin/ inteligencia-emocional-20-anos-de-investigacion-y-desarrollo.html

Jiménez, A. (2018). Inteligencia emocional. En AEPap (Ed.), Curso de actualización pediatría 2018 (pp. 457-469). Lúa Ediciones. https://www.aepap.org/sites/default/files/457469_inteligencia_emocional.pdf

Morales, C. (2004). Personalidad e inteligencia. Fundamentos en Humanidades, 2(10), 69-86. https://dialnet.unirioja.es/descarga/articulo/2004370.pdf 